\title{
An introduction to major astronomical projects in China and plans for the future
}

\author{
Xiangqun Cui \\ National Astronomical Observatories, \\ Nanjing Institute of Astronomical Optics and Technology, \\ CAS, 188 Bancang Street, Nanjing 210042, P.R. China \\ email: xcui@niaot.ac.cn
}

\begin{abstract}
This report is a general introduction to Chinese major astronomical projects. It includes the ongoing project 'Large Sky Area Multi-Object Fiber Spectroscopic Telescope' (LAMOST), and three major projects which have finished their feasibility study and development of key technologies: Five-hundred-meter Aperture Spherical (radio) Telescope (FAST); Space Solar Telescope (SST); Hard X-ray Modulation Telescope (HXMT). Among them, FAST and HXMT have been approved by government in 2006, and SST is pending for the next five years plan. Besides these major projects, a site survey in the west of China, a plan for developing Antarctic Dome A for astronomy, and a preliminary study of Chinese future giant optical/infrared telescopes are also briefly introduced.
\end{abstract}

Keywords. large ground based telescope, space telescope, site survey, antarctic astronomy

\section{Large Sky Area Multi-Object Fiber Spectroscopic Telescope}

LAMOST is a meridian reflecting Schmidt telescope with an average clear aperture of 4 -meter, and a field of view of $5^{\circ}$. There are 4000 optical fibers on its focal surface to transfer light into the 16 spectrographs. Its main scientific goals are: (1) extragalactic spectroscopic survey large-scale structure of the universe and physics of galaxies; (2) stellar spectroscopic survey for structure of the Galaxy and stellar astrophysics; and (3) cross identification of multi-wave band surveys. The main technical challenges in LAMOST include: (a) active optics for segmented deformable mirrors; and (b) parallel controllable fiber positioning. The site of LAMOST is at Xinglong station, about $180 \mathrm{~km}$ north of Beijing. The observing sky area is $-10^{\circ}$ to $+90^{\circ}$ (total 24,000 square degrees). The spectral resolution is 1000-2000 for low-, and 5000-10000 for medium-resolution spectroscopy. With a spectral resolution of $1 \mathrm{~nm}$, the survey maximum magnitude capability is $20.5 \mathrm{mag}$ in about $1.5 \mathrm{hr}$ integration time. The total budget for LAMOST was US $\$ 30 \mathrm{M}$ in 1997 . Before the end of 2005 , the key technologies such as the active optics and the fiber positioning technology have been tested successfully. Integration of mechanical parts has been completed and installed on their concrete pillars. Manufacturing of 4000 optical fibers has started. Now, most mirror polishing is finished and all 61 mirrors will be ready for assembly before the end of 2006. The enclosure and building will be completed in September of 2006. The first light for a 'small LAMOST' with the partial optical aperture $(2 \mathrm{~m}), 250$ optical fibers, one spectrograph and two CCD cameras will be in 2007, and the first light with full aperture and 4000 optical fibers will be in 2008 .

For further details, see: <http://www.lamost.org/en/>. 


\section{Five-hundred-meter Aperture Spherical (radio) Telescope (FAST)}

The Five hundred meter Aperture Spherical Telescope is an Arecibo type large radio telescope. It is also a Chinese concept for SKA. FAST is proposed to be built in the unique karst area of southwest China. It will be over twice as large as Arecibo coupled with much wider sky coverage. Technically, FAST is not simply a copy of the existing Arecibo telescope but has rather a number of innovations: (1) the proposed main spherical reflector, by conforming to a paraboloid of revolution in real time through actuated active control, enables the realization of both wide bandwidth and full polarization capability while using standard feed design; (2) a feed support system which integrates optical, mechanical and electronic technologies will effectively reduce the cost of the support structure and control system. The reflector is a dish with a diameter of $500 \mathrm{~m}$ and a radius of curvature $300 \mathrm{~m}$. The illuminated aperture is $300 \mathrm{~m}$. The opening angle is $120^{\circ}$. For the sky coverage, the maximum observing zenith angle is $50^{\circ}$. Its working frequencies are $0.1-2 \mathrm{GHz}$, up to C-band, X-band. The pointing accuracy is $4^{\prime}$, and the slewing speed is $10^{\circ}$ per minute. The prototype for the feed, the active panels and a $50 \mathrm{~m}$ prototype of FAST have been finished, and some experiments have been successfully done during recent years. The FAST project has passed review by the Chinese Academy of Sciences in 2005 , and was approved by the Chinese government in 2006. The total budget of FAST is US\$85M, and is planned to be completed in 2013.

For further details, see: <http://www.bao.ac.cn/bao/LT/>.

\section{Space Solar Telescope (SST)}

The Space Solar Telescope is proposed to take the advantage of rocketry and abundant research work in solar magnetic field and velocity field in China. SST is a $1 \mathrm{~m}$ solar space telescope with diffraction limited optical images, equipped with an innovative 2-d spectrograph, a $0^{\prime \prime} .5$ resolution soft X-ray telescope, and wide band spectrometer. It will be used in the wide band, continuing evolution observation. With its main character of high resolution, and concentrated in detecting the magnetic elements, the study and observation in the instant and stable magnetic hydrokinetics will take place. SST is waiting for getting funds in the next five years plan (2011-2015).

For further details, see: 〈http://www.bao.ac.cn/bao/SST $>$.

\section{Hard X-ray Modulation Telescope (HXMT)}

The Hard X-ray Modulation Telescope has been selected recently as the first Chinese space astronomy mission for launch in 2010 . The total budget is about US\$80M. Its main scientific goals are deep hard X-ray all-sky survey between $20-250 \mathrm{keV}$ to discover about 1000 new supermassive black holes, pointed observations of faint objects: high-sensitivity timing studies, and high-sensitivity detection of other hard X-ray source. The characteristics are:

- main detector $\mathrm{NaI}(\mathrm{Tl}) / \mathrm{CsI}(\mathrm{Na})$ Phoswich

- total detector area $5000 \mathrm{~cm}^{2}$

- energy range $20-250 \mathrm{keV}$

- energy resolution $22 \%(60 \mathrm{keV})$

- continuum sensitivity $3.0 \times 10^{-7} \mathrm{ph} \mathrm{cm}^{-2} \mathrm{~s}^{-1} \mathrm{keV}^{-1}\left(3 \mathrm{sec}, 100 \mathrm{keV}, 10^{5} \mathrm{~s}\right)$

- field of view $5^{\circ} .7 \times 5^{\circ} .7(\mathrm{FWHM})$

- source location $1^{\prime}(20 \mathrm{~s})$

- angular resolution $5^{\prime}(20 \mathrm{~s})$ 
- mass $\sim 1100 \mathrm{~kg}$, payload $\sim 700 \mathrm{~kg}$

- dimensions $1.7 \times 1.7 \times 1.2 \mathrm{~m}$

- nominal mission lifetime 2 years

- orbit altitude $550 \mathrm{~km}$; inclination $43^{\circ}$

- three-axis stabilized

- control precision $0^{\circ} .25$

- stability $0^{\circ} .005 / \mathrm{s}$

- measurement accuracy $<0^{\circ} .01$

A laboratory prototype has been successfully developed and tested over the last several years. Possible secondary instruments through international collaborations could be a soft $\mathrm{X}$-ray telescope and a wide field X-ray monitor.

For further details, see: <http://www.hxmt.org/english>.

\section{Site survey in west of China}

Western China is a vast area. Most places of this area are at high altitude, with clean air, low temperature, dry environment, less cloudy and dark sky. Targeting on the sites for ELT (optical-IR-submm), National Astronomical Observatories, CAS has started a site survey in the western of China (Tibet, Xinjiang, Qinghai, Yunnan) and set up several stations in 2005 .

\section{Plan for Antarctic Dome A}

A Chinese expedition team arrived at Dome A on Jan. 10, 2005, starting Chinese astronomical activities involved in PANDA - a Chinese key international program for IPY. Some efforts have been done together with the international astronomical community for preparing a site survey at Dome A and studying several wide field telescope plans.

\section{Preliminary study for Extremely Large Telescopes}

Several configurations for extremely large optical/infrared telescope (CFGT) have been studied (Su et al. SPIE Vol.4004, 2000; Su et al. SPIE Vol. 5489, 2004; Su et al. CAA, $28,2004)$, and two configurations for extremely large wide field telescopes have been put forward. For CFGT: (1) one primary focus with FOV $100 \%$ energy concentrated in diameter (d100) in $0^{\prime \prime} .36$ in FOV 20', 4 Nasmyth foci with d100 $0^{\prime \prime} .086$ in FOV $8^{\prime}$ (diffraction limited in FOV $2^{\prime} .83$ ), one Cassegrain focus with d100 $0^{\prime \prime} .11$ in FOV $8^{\prime}$ (diffraction limited in FOV $2^{\prime} .17$ ), one Coudé focus with FOV 29".59 (diffraction limited); (2) all with R-C system; (3) aspherical primary mirror with F/1.2 and 1122 partial annular submirrors; (4) one secondary mirror with diameter $2.476 \mathrm{~m}$ for all optical systems. For the extremely large wide field telescope, two configurations have been studied: (1) LAMOST type extremely large wide field telescope; and (2) a corrector array extremely large wide field telescope. For both cases, all mirrors are plano and spherical. 